\title{
Advantages of Sirolimus in a Calcineurin-Inhibitor Minimization Protocol for the Immunosuppressive Management of Kidney Allograft Recipients
}

\author{
Elias A. Rahal, ${ }^{1}$ Marita Chakhtoura, ${ }^{1}$ Rana Abu Dargham, ${ }^{2}$ Raja B. Khauli, ${ }^{2}$ \\ Walid Medawar, ${ }^{3}$ and Alexander M. Abdelnoor ${ }^{1}$ \\ ${ }^{1}$ Department of Microbiology and Immunology, Faculty of Medicine, American University of Beirut, 11072020 Beirut, Lebanon \\ ${ }^{2}$ Department of Surgery, Faculty of Medicine, American University of Beirut, 11072020 Beirut, Lebanon \\ ${ }^{3}$ Department of Internal Medicine, Faculty of Medicine, American University of Beirut, 11072020 Beirut, Lebanon
}

Correspondence should be addressed to Raja B. Khauli, rkhauli@aub.edu.lb and Alexander M. Abdelnoor, aanoor@aub.edu.lb

Received 5 May 2011; Accepted 25 June 2011

Academic Editors: T. Koshiba, M. Muro, and E. H. Weiss

Copyright ( $) 2011$ Elias A. Rahal et al. This is an open access article distributed under the Creative Commons Attribution License, which permits unrestricted use, distribution, and reproduction in any medium, provided the original work is properly cited.

A myriad of immunosuppressive agents is currently available for the management of graft recipients; however, a consensus on the optimum immunopharmacological plan is nonextant. Twenty kidney recipients on quadruple (mycophenolate mofetil, prednisone, cyclosporine A or tacrolimus, and sirolimus) therapy and 85 on triple therapy where sirolimus was excluded were analyzed for graft rejection or loss within a posttransplant surveillance period of 3 years. Only 1 of 20 recipients (5\%) on quadruple therapy experienced a rejection episode. On the other hand, 13 of 85 recipients (15.3\%) on triple therapy had a rejection episode or lost the graft. Overall, 14 of 105 recipients (13.3\%) experienced a rejection episode or kidney loss. Our observations indicate that an immunosuppressive regimen including sirolimus is advantageous for the management of kidney allograft recipients in the short term.

\section{Introduction}

The pharmacological management mainstay of a renal transplant recipient consists of immunosuppressive agents. The aim of administering these agents is to prevent rejection reactions and to enhance the longevity of the grafted organ. Renal transplantation is the preferred treatment of stage 5 chronic kidney disease (CKD), formerly referred to as endstage renal disease (ESRD) [1].

Administered immunosuppressive regimens often include two phases. The initial phase, induction therapy, consists of intense immunosuppression during the first few days following transplantation. The second phase, maintenance therapy, includes long-term administration of immunosuppressive agents at relatively low doses. In case of a rejection reaction, a transplant recipient may be administered additional immunosuppressive agents [2].

Induction and maintenance regimen often includes corticosteroids, such as prednisone, hydrocortisone, or methylprednisolone. These have suppressive effects on both humoral and cellular immune mechanisms; however, corticosteroids are associated with a myriad of side effects including hyperlipidemia, osteoporosis, glucose intolerance, vascular disease, and hypertension [3]. Agents administered also include the immunophilin-binding calcineurin inhibitors, cyclosporine $\mathrm{A}$, and tacrolimus, which decrease IL-2 production and limit T-cell activation. Although these agents improve short-term outcomes by preventing rejection reactions, they are attributed with considerable side effects that encompass vasculopathy and nephrotoxicity in the long term [4]. Sirolimus, a more novel agent, inhibits the mammalian target of rapamycin (mTOR) leading to inhibition of lymphocyte growth and differentiation. It is less nephrotoxic but is associated with suppressive effects on the bone marrow, decreased wound healing, and hyperlipidemia [5]. Mycophenolate mofetil (MMF), on the other hand, inhibits inosine monophosphate dehydrogenase thus limiting de novo purine nucleotide synthesis. Inhibition of this pathway primarily impacts lymphocytes. MMF is not 
TABLE 1: Effect of donor type on graft rejection or loss.

\begin{tabular}{lccc}
\hline Donor type & Total cases & $\begin{array}{c}\text { Rejection } \\
\text { or graft } \\
\text { loss cases }\end{array}$ & $\begin{array}{c}\text { Percent rejection or } \\
\text { graft loss }\end{array}$ \\
\hline $\begin{array}{l}\text { Living-related donor } \\
\begin{array}{l}\text { Living-unrelated } \\
\text { donor }\end{array}\end{array}$ & 45 & 5 & $11.1 \%$ \\
Cadaveric donor & 2 & 8 & $13.3 \%$ \\
\hline
\end{tabular}

associated with nephrotoxicity, hyperlipidemia, or vascular disease; its adverse effects, however, include gastrotoxicity [6]. Other agents include polyclonal and monoclonal antibodies with various cellular and molecular targets in addition to antimetabolites that affect cellular processes.

The use of therapeutic regimens that include a combination of immunosuppressive agents having different mechanisms of action permits the use of lower doses of each and thus reduces the occurrence of toxic side effects. As of yet there is no consensus on the use of a drug combination that would yield optimum results. In this stud, we compared the effects of 2 regimens given to kidney recipients on rejection or loss of graft. One regimen comprised a combination of mycophenolate mofetil, prednisone, cyclosporine A or tacroliamus, and sirolimus (quadruple therapy), and the other excluded sirolimus (triple therapy).

\section{Materials and Methods}

2.1. Kidney Transplant Recipients. One hundred and five kidney allograft recipients were included in the study. Forty five received a graft from a living-related donor, while 58 received a kidney from a living-unrelated donor. Two recipients received a cadaveric graft. The study population comprised 29 females and 76 males. The mean age was 44.6 (Std. deviation 15.2) with the youngest recipient being 6 years of age and the oldest 73. Rejection or loss of the graft within a maximum period of 3 years following transplantation was recorded (Table 1).

2.2. Immunosuppressive Agents. Induction immunosuppressive regimens consisted of triple immunosuppression with mycophenolate mofetil (MMF), prednisone (PRED), and cyclosporin A or tacrolimus (TACRO). As part of such a regimen, MMF was given at a dose of $1000 \mathrm{mg}$ twice daily, a PRED taper of $60-30 \mathrm{mg}$ over 8 days was administered, and CYCLO was given at a dose of $8-12 \mathrm{mg} / \mathrm{kg} /$ day. On the other hand, TACRO was given at a dose of 2-3 mg per day. Induction therapy employing a quadruple immunosuppressive plan included MMF, PRED, CYCLO, and sirolimus (SIRO). In this plan, MMF was given as a dose of 250-500 mg twice per day, a PRED taper of 60-25 mg was administered over 8 , and CYCLO was given at a dose of 3-6 mg/kg/day. On the other hand, SIRO was given at a dose of $4 \mathrm{mg}$ on the first day after operation and as $2 \mathrm{mg}$ per day afterward. Patients in both groups additionally received a bolus or short course of antithymocyte globulin $(4-6 \mathrm{mg} / \mathrm{kg})$.
TABLE 2: Effect of immunosuppressive regimen on graft rejection or loss.

\begin{tabular}{lccc}
\hline $\begin{array}{l}\text { Immunosuppressive } \\
\text { regimen* }\end{array}$ & Total cases & $\begin{array}{c}\text { Rejection } \\
\text { or graft } \\
\text { loss cases }\end{array}$ & $\begin{array}{c}\text { Percent rejection } \\
\text { or graft loss }\end{array}$ \\
\hline MMF/Pred/Cyclo & 74 & 10 & $13.5 \%$ \\
MMF/Pred/Tacro & 11 & 3 & $27.3 \%$ \\
MMF/Pred/Cyclo/Siro & 20 & 1 & $5.0 \%$ \\
\hline *MMF: mycophenolate mofetil, PRED: prednisone, CYCLO: cyclosporin A, \\
TACRO: tacrolimus, SIRO: sirolimus.
\end{tabular}

Doses used for maintenance immunosuppression included 500-1000 mg of MMF per day, $10 \mathrm{mg}$ of PRED per day, and 3-5 mg/kg/day of CYCLO with a trough level target of $200-300 \mathrm{ng} / \mathrm{mL}$ during the first 6 months following the operation and 100-150 ng/mL afterward. When used instead of CYCLO, TACRO was given at a dose of $2 \mathrm{mg}$ per day and SIRO at a dose of $2 \mathrm{mg}$ per day.

Twenty recipients were given quadruple therapy, and 85 were given triple therapy.

\section{Results}

3.1. Effect of Transplant Type on Graft Rejection or Loss. Five of 45 patients (11.1\%) who received a kidney from a livingrelated donor suffered a rejection reaction or loss of the graft. On the other hand, 9 (15.2\%) of 59 patients who received a kidney from a living-unrelated donor or cadaveric donor suffered rejection or loss of the transplanted organ. Overall, 14 rejection or graft loss cases occurred (Table 1 ).

\subsection{Effect of Immunosuppressive Regimen on Graft Rejection} or Loss. In the group receiving triple immunosuppressive treatment, 10 of 74 patients $(13.5 \%)$ on a regimen of MMF/PRED/CYCLO had a rejection episode or lost the graft. On the other hand, $3(27.3 \%)$ of 11 recipients on triple immunosuppression with MMF/PRED/TACRO suffered graft rejection or loss. In the group receiving quadruple immunosuppressive therapy, 1 (5\%) of 20 patients on a regimen of MMF/PRED/CYCLO/SIRO had a rejection reaction (Table 2 ).

\section{Discussion}

An improvement in transplantation short-term outcomes, including minimization of rejection risk, during recent years is generally attributed to advances in immunosuppressive regimens. However, long-term graft survival is not believed to have greatly improved despite these advances [7]. This may be due to the toxicities of the employed drugs themselves or due to the immunosuppressive predispositions imposed by these drugs. Although steroids and calcineurin inhibitors remain the most widely prescribed agents to transplant recipients, their toxicities have led to a current trend of minimizing their doses concomitant with implementation of more novel and less toxic agents $[8,9]$. Despite the myriad of immunosuppressive regimen examined by the literature, 
a lack of consensus on the most beneficial protocol remains prevalent. Here, we relay our experience with the effects of various immunosuppressive protocols on short-term kidney allograft survival.

There was no significant difference in survival between grafts obtained from living-related and living non-related donors. Having ruled out the confounding effects of graft source, we analyzed our patient dataset for the effect of immunosuppressive treatment regimen on graft rejection or loss.

Both cyclosporine A and tacrolimus are calcineurin inhibitors. However, in the triple therapy regimen, the former agent appears to be superior in preventing rejection episodes or graft loss. Rejection episodes or graft loss was twice as much when tacrolimus was used instead of cyclosporine A.

The regimen to yield the lowest percent $(5 \%)$ of rejection reactions or graft loss was a quadruple immunosuppressive protocol. This directed our attention to sirolimus which has garnered interest in recent years as a potent agent whose implementation may be more advantageous than other drugs. Multiple reports including ones by members of our group $[10,11]$ have indicated the benefits of implementing sirolimus in the management of transplant recipients. This agent not only has suppressive effects on cell-mediated immunity, but also it appears to inhibit antibody production after transplantation [12-14]. Terasaki and Ozawa [15, 16] have previously reported that $21 \%$ of graft recipients produce de novo HLA antibodies and that this population has an increased graft loss rate. In their studies, subjects on cyclosporine A and mycophenolate mofetil had lower frequencies of HLA antibodies. As previously reported, sirolimus also appeared to suppress the de novo production of anti-HLA antibodies [11]. This may explain the decreased rate of graft rejection or loss in our population dataset; however, this requires further investigation. Therefore, our results highlight the relevance of this drug and concur with reports indicating the advantages of its implementation as part of an immunosuppressive regimen for renal allograft recipients.

\section{Conclusions}

The ultimate aim of posttransplant therapy is to find a balance between immunosuppression and minimization of drug side effects while enhancing the longevity of the graft. Our findings indicate that a quadruple immunosuppressive regimen consisting of mycophenolate mofetil, prednisone, cyclosporin A, and sirolimus may be the optimum regimen when examining short-term outcomes. The long-term effects of this regimen and whether it offers unprecedented advantages remain to be seen.

\section{Acknowledgments}

The authors wish to thank Ms. Aline Yacoubian for her assistance. This work was funded by the Lebanese National
Council for Scientific Research (CNRS). A. M. Abdelnoor, R. B. Khauli and E. A. Rahal contributed equally to this paper.

\section{References}

[1] A. S. Levey, J. Coresh, K. Bolton et al., "K/DOQI clinical practice guidelines for chronic kidney disease: evaluation, classification, and stratification," American Journal of Kidney Diseases, vol. 39, no. 2, pp. S1-S266, 2002.

[2] P. F. Halloran, "Immunosuppressive drugs for kidney transplantation," The New England Journal of Medicine, vol. 351, no. 26, pp. 2715-2729, 2004.

[3] F. Citterio, "Steroid side effects and their impact on transplantation outcome," Transplantation, vol. 72, no. 12, pp. SS75SS80, 2001.

[4] M. Naesens, D. R. J. Kuypers, and M. Sarwal, "Calcineurin inhibitor nephrotoxicity," Clinical Journal of the American Society of Nephrology, vol. 4, no. 2, pp. 481-508, 2009.

[5] P. Dupont and A. N. Warrens, "The evolving role of sirolimus in renal transplantation," QJM, vol. 96, no. 6, pp. 401-409, 2003.

[6] A. N. Warrens, "The evolving role of mycophenolate mofetil in renal transplantation," QJM, vol. 93, no. 1, pp. 15-20, 2000.

[7] R. Marcn, "Immunosuppressive drugs in kidney transplantation: impact on patient survival, and incidence of cardiovascular disease, malignancy and infection," Drugs, vol. 69, no. 16, pp. 2227-2243, 2009.

[8] A. J. Matas, D. Granger, D. B. Kaufman et al., "Steroid minimization for sirolimus-treated renal transplant recipients," Clinical Transplantation, vol. 25, no. 3, pp. 457-467, 2011.

[9] S. M. Flechner, "Minimizing calcineurin inhibitor drugs in renal transplantation," Transplantation Proceedings, vol. 35, no. 3, pp. 118S-121S, 2003.

[10] R. B. Khauli, A. Houjaij, S. Sawah et al., "Observations on quadruple immunosuppression maintenance therapy using rapamycin, low-dose cyclosporine, mycophenolate mofetil, and prednisone following ATG induction," Transplantation Proceedings, vol. 37, no. 7, pp. 3031-3033, 2005.

[11] A. M. Abdelnoor, R. Ajib, M. Chakhtoura et al., "Influence of HLA disparity, immunosuppressive regimen used, and type of kidney allograft on production of anti-HLA classI antibodies after transplant and occurrence of rejection," Immunopharmacology and Immunotoxicology, vol. 31, no. 1, pp. 83-87, 2009.

[12] H. Chen, H. Luo, P. Daloze et al., "Long-term in vivo effects of Rapamycin on humoral and cellular immune responses in the rat," Immunobiology, vol. 188, no. 3, pp. 303-315, 1993.

[13] M. D. Pescovitz, B. K. Book, S. Henson et al., "The addition of sirolimus to cyclosporine and steroids inhibits the anti-equine antibody response in renal transplant recipients treated with antithymocyte globulin," American Journal of Transplantation, vol. 3, no. 4, pp. 497-500, 2003.

[14] D. J. Propper, J. Woo, A. M. Macleod, G. R. D. Catto, and A. W. Thomson, "The effects of rapamycin on humoral immunity in vivo: suppression of primary responses but not of ongoing alloantibody synthesis or memory responses," Transplantation, vol. 54, no. 6, pp. 1058-1063, 1992.

[15] P. I. Terasaki and M. Ozawa, "Predicting kidney graft failure by HLA antibodies: a prospective trial," American Journal of Transplantation, vol. 4, no. 3, pp. 438-443, 2004.

[16] P. I. Terasaki and M. Ozawa, "Predictive value of HLA antibodies and serum creatinine in chronic rejection: results of a 2-year prospective trial," Transplantation, vol. 80, no. 9, pp. 1194-1197, 2005. 


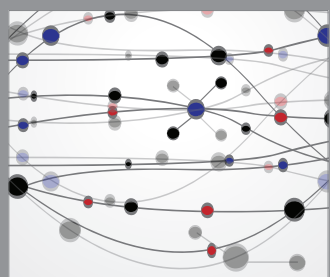

The Scientific World Journal
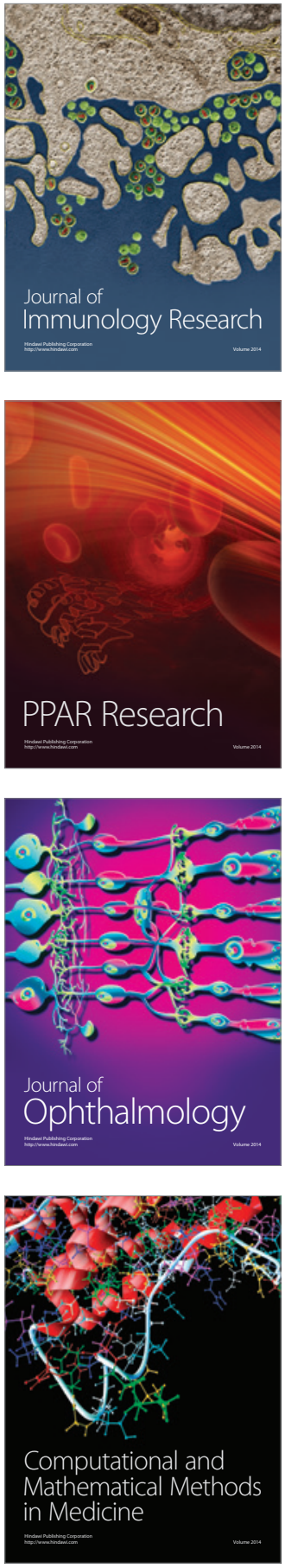

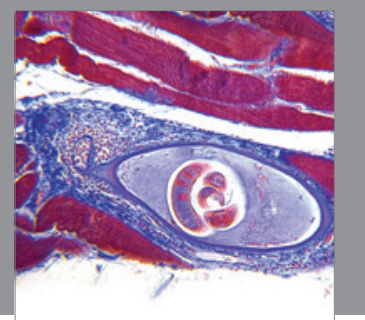

Gastroenterology

Research and Practice
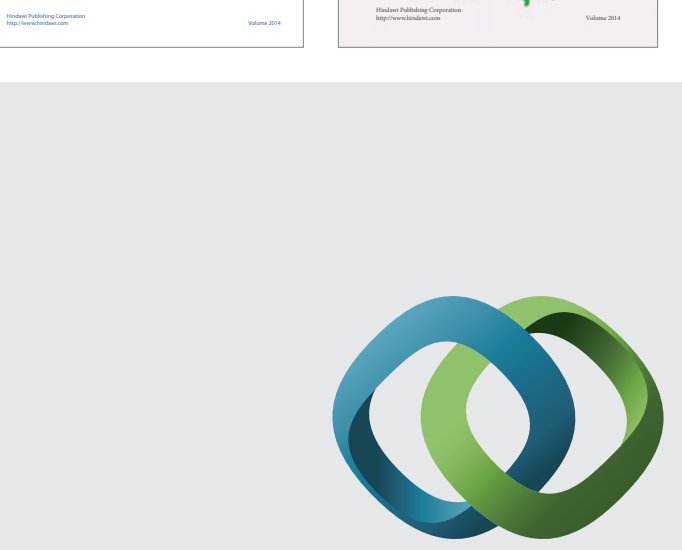

\section{Hindawi}

Submit your manuscripts at

http://www.hindawi.com
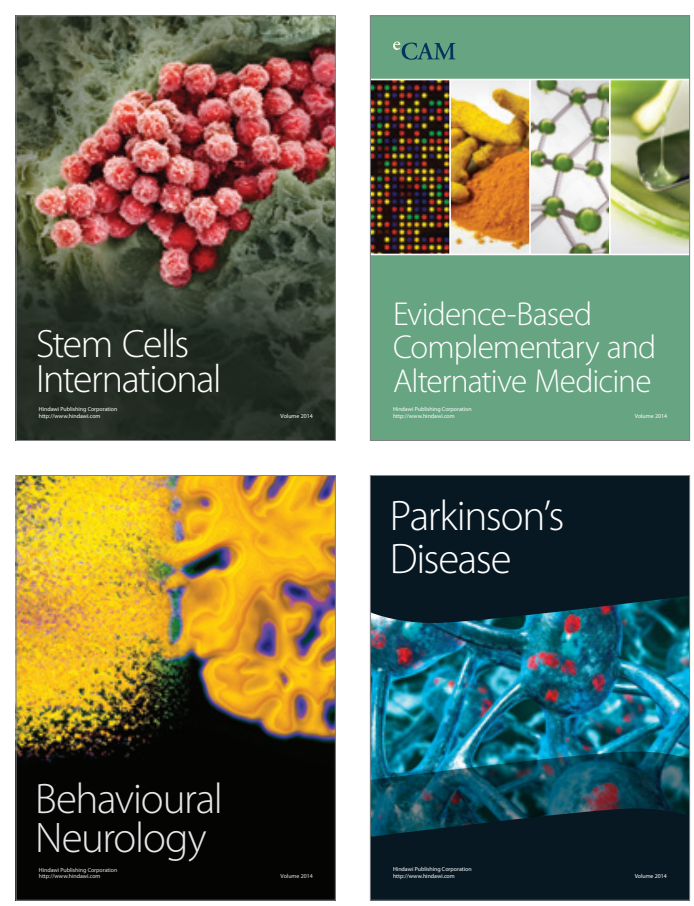

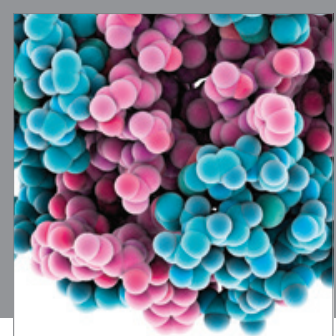

Journal of
Diabetes Research

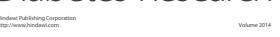

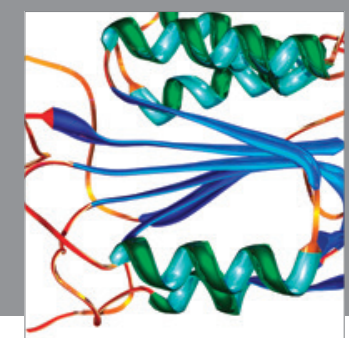

Disease Markers
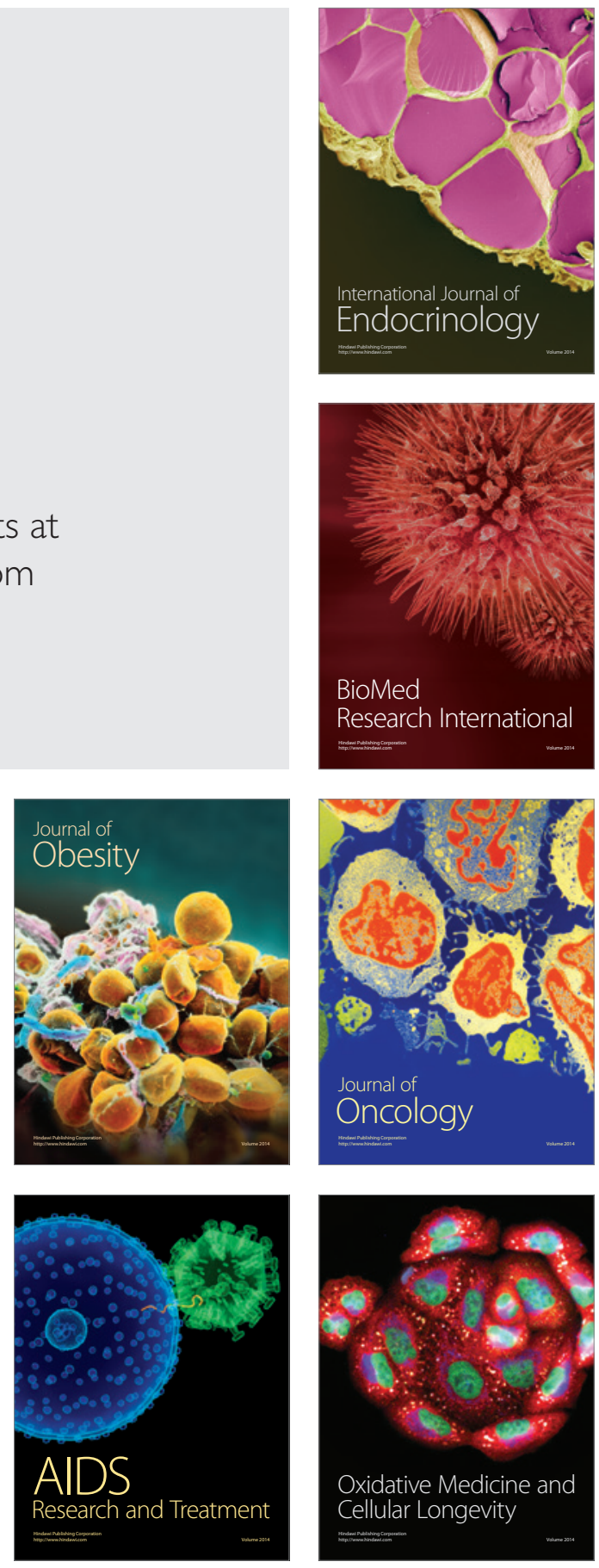